\title{
Contributions of Mainstream Sexual Media Exposure to Sexual Attitudes, Perceived Peer Norms, and Sexual Behavior: A Meta- Analysis
}

Sarah M. Coyne

Brigham Young University - Provo, smcoyne@byu.edu

L. Monique Ward

University of Michigan - Ann Arbor

Savannah L. Kroff

Brigham Young University - Provo

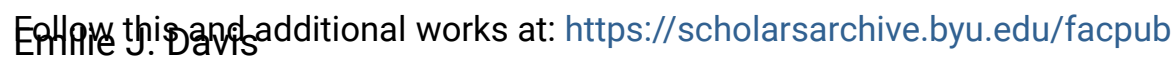

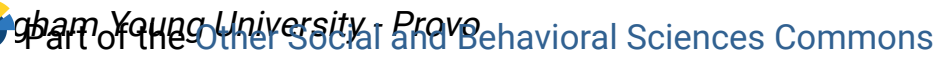

Hailey G. Holmgren

\section{Briggimal ßBubdicatiersifyitation}

Coyne, S.M., Ward, L.M., *Kroff, S.L., *Davis, E.J., *Holmgren, H.G., Jensen, A. C., Erickson, S., \& *Essig, L.W. (2019). Contributions of mainstream sexual media exposure to sexual attitudes, See next page for additional authors perceived peer norms, and sexual behavior: A meta-analysis. Journal of Adolescent Health, 64, 430-436.

\section{BYU ScholarsArchive Citation}

Coyne, Sarah M.; Ward, L. Monique; Kroff, Savannah L.; Davis, Emilie J.; Holmgren, Hailey G.; Jensen, Alexander C.; Erickson, Sarah E.; and Essig, Lee W., "Contributions of Mainstream Sexual Media Exposure to Sexual Attitudes, Perceived Peer Norms, and Sexual Behavior: A Meta-Analysis" (2019). Faculty Publications. 4131.

https://scholarsarchive.byu.edu/facpub/4131

This Peer-Reviewed Article is brought to you for free and open access by BYU ScholarsArchive. It has been accepted for inclusion in Faculty Publications by an authorized administrator of BYU ScholarsArchive. For more information, please contact ellen_amatangelo@byu.edu. 


\section{Authors}

Sarah M. Coyne, L. Monique Ward, Savannah L. Kroff, Emilie J. Davis, Hailey G. Holmgren, Alexander C. Jensen, Sarah E. Erickson, and Lee W. Essig 
Review article

\title{
Contributions of Mainstream Sexual Media Exposure to Sexual Attitudes, Perceived Peer Norms, and Sexual Behavior: A Meta-Analysis
}

\author{
Sarah M. Coyne, Ph.D. ${ }^{a, *}$, L. Monique Ward, Ph.D. ${ }^{b}$, Savannah L. Kroff, M.S. ', Emilie J. Davis, M.S. ${ }^{a}$, \\ Hailey G. Holmgren, M.S. a , Alexander C. Jensen, Ph.D. a , Sarah E. Erickson, Ph.D. ', and
} Lee W. Essig, M.S. ${ }^{\text {a }}$

${ }^{a}$ School of Family Life, Brigham Young University, Provo, Utah
${ }^{\mathrm{b}}$ Department of Psychology, University of Michigan, Ann Arbor, Michigan
${ }^{\mathrm{c}}$ Communications Department, University of Michigan, Ann Arbor, Michigan

Article history: Received July 18, 2018; Accepted November 14, 2018

Keywords: Sex; Media; Meta-analysis; Television; Music; Video games; Film

\section{A B S T R A C T}

Purpose: Decades of research have examined the impact of exposure to nonexplicit portrayals of sexual content in media. There is only one meta-analysis on this topic, which suggests that exposure to "sexy media" has little to no effect on sexual behavior. There are a number of limitations to the existing meta-analysis, and the purpose of this updated meta-analysis was to examine associations between exposure to sexual media and users' attitudes and sexual behavior. Methods: A thorough literature search was conducted to find relevant articles. Each study was coded for associations between exposure to sexual media and one of six outcomes including sexual attitudes (permissive attitudes, peer norms, and rape myths) and sexual behaviors (general sexual behavior, age of sexual initiation, and risky sexual behavior).

Results: Results from 59 studies, involving 394 effect sizes, revealed that exposure to sexual media had a small but significant effect on both sexual attitudes and behaviors; the effect size was comparable to other media effects meta-analyses. Effects were stronger for adolescents than emerging adults. In addition, effects were stronger for boys than girls and for white participants compared with black participants.

Conclusion: This study suggests that exposure to nonexplicit sexual media is associated with both sexual attitudes and behavior, particularly during adolescence. Implications for parents, media producers, and researchers are discussed.

(c) 2018 Society for Adolescent Health and Medicine. All rights reserved.

IMPLICATIONS AND
CONTRIBUTION
This meta-analysis sug-
gests that exposure to
nonexplicit sex in media is
associated with sexual
attitudes and behaviors,
especially during
adolescence.

Sexual content is a staple in mainstream American media. We define sexual content as verbal or visual references to sexual relationships, courtship, or sexual acts; mainstream American

\footnotetext{
Conflicts of interest: The authors have no conflicts of interest to disclose. Current address for Sarah E. Erickson is Communication Department at Trinity University, San Antonio, Texas.

* Address correspondence to: Sarah M. Coyne, Ph.D., School of Family Life, Brigham Young University, JFSB 2086C, Provo, UT 84602.

E-mail address: smcoyne@byu.edu (S.M. Coyne).
}

media are defined as mass media targeting all age groups (i.e., not sexually explicit media/pornography). Analyses estimate that sexual content appears in $81 \%$ of major motion pictures [1] and $82 \%$ of television programs [2]. Although sexual content is prevalent, it is not uniform and instead features some themes more than others. Content analyses indicate a heavy emphasis on sex as casual, relatively risk-free, and as following strict gender scripts [3]. Based on these characteristics, researchers have questioned how frequent exposure to this content might be 
shaping the sexual attitudes, expectations, and behaviors of adolescents and emerging adults. Existing analyses have produced somewhat inconsistent results and also document contributions of several moderating factors (e.g., participant gender and race). The current meta-analysis represents one of the first attempts to provide a statistical review of the strength of findings in this field [4].

Multiple theories and mechanisms support a potential role of media use. Cultivation theory [5] focuses on exposure levels as the mechanism, arguing that repeated exposure to commonly portrayed media messages will foster analogous beliefs in media users. Social cognitive theory [6] centers observational learning and related cognitions as the mechanisms, arguing that sexual behaviors can be shaped by observing, identifying with, and following the rewarded actions of media models. Finally, with a focus on our learning of the culture's sexual scripts, Wright's acquisition, activation, application model of media sexual socialization ( ${ }_{3} \mathrm{AM}$ ) [7] argues that sexual media content exposes consumers to novel sexual scripts (acquisition), primes existing sexual scripts (activation), and is used to guide one's own sexual activities or judgments of other's activities (application). Together, these theories suggest that sexual media could shape sexual beliefs, behaviors, and adherence to cultural scripts, and we therefore investigate all three outcomes here.

\section{Empirical evidence of contributions of media exposure}

Three questions about potential effects have frequently been raised regarding exposure to the sexual content of mainstream media. One question concerns the impact of these high prevalence levels: Does frequent exposure to sexual media influence users' expectations of other's sexual behavior or affect their own sexual decisions? Several studies have found that greater sexual media exposure is linked to higher estimates of other people's sexual experiences [8] and is also related to earlier sexual initiation or more extensive levels of sexual experience. [9-11]. Conversely, other studies have found that sexual media exposure does not influence viewers' sexual expectations or behavior [12-14].

A second question centers on prominent depictions of sex as casual and risk-free. Is heavy exposure to sexual media content, which frequently conveys this theme, related to holding more permissive sexual attitudes? Sexual permissiveness is defined as the acceptability of premarital sex, casual sex, or uncommitted sexual exploration [15]. This question has also been applied to sexual behavior, with investigators testing whether exposure to risk-free sexual content encourages greater levels of sexual risktaking. Several studies suggest that exposure to sexual media is associated with higher levels of unprotected sex, unplanned pregnancies, sexually transmitted infections, and one-night stands $[16,17]$.

A third question concerns potential effects of the media's stereotypical portrayals of sexual roles for women and men. In mainstream media, male sexuality is often portrayed as active, powerful, and persistent, and female sexuality as passive, appearance-based, and partner-pleasing. This pattern corresponds to the culture's dominant courtship script labeled the heterosexual script [18]. Because this script posits an adversarial relationship between the sexes, one in which women are sexually objectified (and therefore dehumanized), and male pleasure is prioritized, the concern is that these dynamics could help legitimize sexual violence against women [19]. Evidence indeed indicates that heavier media use is associated with a greater acceptance of myths about rape $[20,21]$.

\section{Questions remaining and inconsistent results}

Despite the significant findings reported previously, results are not consistent. Moreover, contributions of media use to sexual outcomes are sometimes affected by moderators, including media genre, and characteristics of the viewer such as gender and race. For example, some report that higher levels of media use are associated with more permissive sexual attitudes for only female [22] or only male [23] participants. Other studies report strong media effects for white participants, but few effects among black participants [24,25].

There is one meta-analysis, to our knowledge, addressing the topic of sexual media and outcomes. Ferguson et al. [4] examined this topic in 22 correlational and longitudinal studies and found that general media use did not correlate with sexual behaviors. In addition, they found that sexual media use was only weakly correlated with sexual behaviors $(r=.08)$, after controlling for various factors, including family and peer influences. This meta-analysis would suggest that exposure to nonexplicit sexual media has little or no impact on sexual outcomes.

An examination of this meta-analysis leaves the reader with significant questions. There are few moderators examined, apart from whether the study controlled for family or peer environment. However, the research literature suggests several important moderators, such as age, ethnicity, media type, and study design that were excluded from analysis. Notably, the analysis excluded all experimental studies. There was no explanation for this choice, but this decision omits several studies that use a methodology that allows the researcher to control several extraneous factors. In addition, the analysis only included studies that reported controlled effects sizes (i.e., beta coefficients). This technique is controversial in the meta-analytic field (see commentary by several scholars [26,27]) and may exclude many relevant studies. Indeed, the search revealed only 22 relevant studies, of which only 14 studies included sexual media as an independent variable. This limited sample size is surprising, given the rich literature on sexual media effects. In addition, the metaanalysis did not include any studies that examined sexual attitudes, which, according to our literature review, is a substantial omission. Furthermore, the meta-analysis focused only on adolescents. Although some adolescents engage in sexual activity, many individuals wait until emerging adulthood before becoming sexually active [28]. The analysis therefore likely excludes much sexual activity occurring among young people. These issues and other concerns led us to conduct our own meta-analysis on outcomes of exposure to nonexplicit sexual media.

Accordingly, our goal was to use meta-analysis to provide a statistical review of the state of findings concerning effects of exposure to sexual media. For sexual outcomes, we focused on sexual attitudes (i.e., permissive sexual attitudes, expectations of peer norms, and rape myth acceptance) and sexual behavior (i.e., general level of sexual activity, risky sex, and age of sexual initiation). We also investigated several media formats, testing contributions of television, music videos, movies, video games, and music lyrics. Finally, we examined 
multiple potential moderators including participant age, gender, and ethnicity, as each has been shown to moderate sexual media effects.

\section{Methods}

\section{Literature search procedures}

From January through April 2015, MEDLINE, PsycINFO, and Communications and Mass Media were used to search for relevant articles. The following terms were used: "sexual attitude," "permissive attitude," "peer norm," "rape myth," and "sexual behavior," "sexual initiation," "risky sex," "sexual intercourse," and "media," "television," "movie," "music," "music videos," and "video games." The reviews on sexual media by Ward [29] and Ward et al. [30] were used to locate additional articles. In addition, we contacted any author publishing a minimum of two studies on sex and media to request raw data or unpublished articles. Another search was conducted using these criteria in January 2018, yielding three possibly relevant studies, one of which met appropriate inclusionary and exclusionary criteria. The search was not limited by year of publication.

From these searches, 3,933 articles were found. Coders read the title and abstract (and often the full text of the article) to decide on relevancy. Disagreements were extremely rare and were decided by a moderator if needed. Of these articles, 200 were found as possibly applicable to the study (see Figure 1). One hundred and thirteen articles were excluded for not measuring the outcomes relevant to the present study. An additional 28 studies were excluded for not containing the necessary statistics (e.g., qualitative studies, studies where only unstandardized betas were provided). Where applicable, authors were contacted in efforts to

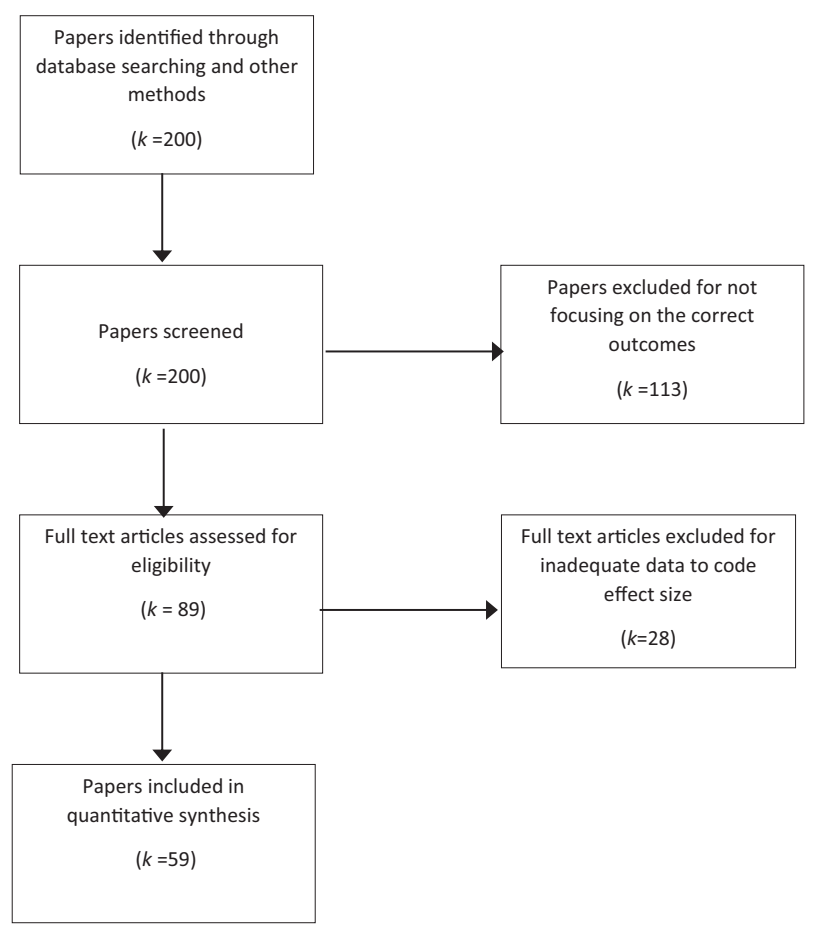

Figure 1. Flowchart for search procedures. procure the necessary statistics before the study was excluded. Together, these efforts left a total of 59 studies for analysis.

\section{Inclusion and exclusion criteria}

Articles were determined to be fit for use based on several inclusionary criteria. First, studies had to measure effects or associations of sexual media on either sexual attitudes or sexual behaviors. Many studies overtly measured sex in media (including many of the experimental and some of the correlational studies). However, many studies examined the impact of media on sexual outcomes, but it was not clear whether sexual content was actually consumed by viewers. For example, some studies measured overall media time (which could include any type of content) or specified genre (e.g., drama, comedy, sports, and music), which may or may not include sexual content depending on the program viewed. Accordingly, we included studies with a general media variable (reflecting media content that was not necessarily sexual in nature) only if the authors had conducted a preliminary content analysis showing that their specific medium was high in sexual content [15]. Although this a somewhat conservative approach, we feel that this choice adds to the validity of the study by ensuring that the media consumed were of a sexual nature. Second, media exposure had to include one of the following: television, film, video game, music, or music video. Studies were excluded if they focused on other types of media (e.g., books, magazines, and Web sites). Several studies included multiple media types and only provided a combined media measure. We only included these studies if most media types included were from the genres in the inclusion criteria. Studies that focused on explicit portrayals of sex (i.e., pornography) were excluded. Third, studies had to include empirical data. Fourth, studies were only included if they provided enough statistical information to be coded in the meta-analysis. Fifth, studies were excluded if they focused on the health risks or negative outcomes of risky sexual behavior (e.g., sexually transmitted infections). Finally, only studies that were written in or translated into English were included.

\section{Reliability}

Two of the authors examined both the abstracts and text to determine the relevance of each article. The coders identified relevant articles, and one primary author rechecked each article for relevancy. Reliability was first examined by having all coders code $15 \%$ of the entire sample. Cohen's Kappa was then conducted on all study variables. Acceptable reliability was achieved for each variable, namely, gender, $\kappa=$ 1.00 ; age, $\kappa=1.00$; ethnicity, $\kappa=1.00$; study design, $\kappa=.85$; region, $\kappa=.74$; medium viewed, $\kappa=1.00$; attitude, $\kappa=1.00$; and behavior, $\kappa=1.00$. Finally, coders individually coded each relevant article for moderators and outcomes. Disagreements were rare, and coders were required to reach $100 \%$ consensus on all codes.

\section{Coding of studies}

Sexual attitudes and behaviors. Each study was coded for one of six outcomes. For studies that measured sexual attitudes, three outcomes were coded, including (1) permissive attitudes (i.e., one-night stands are totally acceptable), (2) attitudes toward peer 
norms (see Note 1; i.e., kids in my class are all having sex), or (3) rape myth acceptance (i.e., it's okay for a woman to get raped if she is wearing suggestive clothing). For studies that measured sexual behaviors, three outcomes were coded, including (1) sexual initiation (i.e., age at first intercourse), (2) risky sex (e.g., number of partners, one-night stands, and no contraception), or (3) general level of sexual experience (e.g., level of experience with kissing and precursory sexual behaviors).

Sex and age of participant. The sex of each study sample was recorded as male, female, or combined (if the study did not provide statistics separately for men and women). The average age of participant was determined from each relevant article and was placed in one of three categories: childhood (age 0-10 years), adolescence (age 11-17 years), or emerging adult (age 18-25 years).

Region. The region in which each study took place was coded in one of eight categories, based on continent: (1) North America, (2) Europe, (3) Asia, (4) Africa, (5) South America, (6) Australasia, (7) combined, and (8) not specified. If a study had participants from two or more regions, this study was coded as "combined."

Ethnicity. The ethnicity of participants was determined based on the majority ethnicity of the study sample. If $75 \%$ of the sample was one ethnicity, coders would mark one of six categories: (1) white, (2) black, (3) Asian, (4) Hispanic, (5) other, (6) not reported, and (7) multiple groups (selected if there was no $75 \%$ ethnic majority).

Media type. Media type was placed in one of six categories: (1) TV program, (2) video games, (3) music video (4) music, (5) movies/videos, and (6) multiple media. If two or more media were used (e.g., if a study used music and music video as the media type), it was placed under "multiple media."

Study design. Study design was coded as (1) experimental, (2) cross-sectional, or (3) longitudinal.

Other publication features. Year of study and publication status (published or unpublished) were also coded for each study.

\section{Computation of effect sizes}

We obtained Pearson's $r$ directly from each study. Each correlation coefficient underwent a fisher $z$-transformation to normalize its distribution [31]. The average effect size for each outcome was obtained and transformed back into Pearson's $r$ for interpretation. The pooled estimate of $r$ is denoted as $r_{+}$. In the case where the correlation coefficients were not reported ( $k=19$ ), we contacted corresponding authors via e-mail to obtain $r$. If no response from authors was given or no relevant statistics were available $(k=16)$, we used available standardized regression coefficients $(\beta)$ through a deterministic imputation formula $(r=\beta+.05 \lambda$, where $\lambda$ is 1 if $\beta$ is non-negative and 0 if $\beta$ is

Note 1:

This construct focused on participants' beliefs or assumptions about the sexual attitudes and experiences of their peers, including how accepting their peers are of premarital sex, and how sexually experienced their peers are (e.g., estimating percentage of peers who have had sex). negative) [32]. Although this imputation has limitations, it is superior to replacing the missing correlations with zero or with the mean of all the correlations. We ran analyses for transformed compared with untransformed studies to examine whether including the transformed studies noticeably changed the strength of the results. In addition, we used the formula described in Lipsey and Wilson [33] to compute effect sizes for those studies examining standardized mean differences (primarily experiments).

The vast majority of the studies in our meta-analysis reported more than one effect size. Other meta-analyses have either picked one representative effect size from each study [4] or have combined and then averaged effect sizes within studies to provide an overall effect size estimate. However, these methods either result in vast amounts of missing data or violate the assumption of independency of effect sizes, given that the individual effect sizes in any given study are likely related. Accordingly, we used a threelevel random effects model, using the metafor package in $R$. version 3.5.1 (https://cran.r-project.org) [34,35] The three-level model weights effect sizes by sample size and allows for variance between studies, effect sizes from any individual study, and sampling variance. This technique also allows us to examine both within- and between-study heterogeneity while retaining as much data as possible, resulting in a model with high statistical power. If there was evidence of heterogeneity, we conducted a number of moderator analyses. For these analyses, each moderator with $k$ categories was recoded to $k-1$ dummy variables using binary coding.

\section{Results}

The current meta-analysis consisted of 59 studies in 56 articles. Collectively, there were 48,471 participants. Detailed information for each study can be found in the Supplementary Data. Overall, 394 independent effect size estimates were available. See Table 1 for results for major analyses in the study.

Table 1

Overview of results

\begin{tabular}{llll}
\hline Outcome & Effect size $\left(r_{+}\right)$ & CI & $n$ \\
\hline Overall & $.14^{* * *}$ & .11 to .17 & 394 \\
Sexual attitudes & & & \\
$\quad$ Permissive & $.15^{* * *}$ & .10 to .19 & 56 \\
$\quad$ Peer sexual norms & $.13^{* * *}$ & .03 to .23 & 40 \\
$\quad$ Rape myths & $.22^{*}$ & .04 to .40 & 6 \\
Sexual behavior & & & \\
$\quad$ General sexual behavior & $.16^{* * *}$ & .12 to .19 & 123 \\
$\quad$ Risky sexual behavior & $.11^{* * *}$ & .05 to .20 & 115 \\
$\quad$ Age of sexual initiation & $-.16^{* * *}$ & -.06 to -.25 & 54 \\
Age & & & \\
$\quad$ Adolescent & $.18^{* * *}$ & .15 to .21 & 241 \\
$\quad$ Emerging adults & $.06^{* * *}$ & .03 to .10 & 151 \\
Ethnicity & & & \\
$\quad$ White & $.17^{* * *}$ & .12 to .23 & 118 \\
$\quad$ Black & .08 & -.01 to .16 & 19 \\
$\quad$ Hispanic & $.15^{* *}$ & .04 to .25 & 14 \\
Sex of participant & $.18^{* * *}$ & .13 to .23 & 71 \\
$\quad$ Males & $.12^{* * *}$ & .07 to .17 & 71 \\
$\quad$ Females &
\end{tabular}

Only variables that were significant in the moderator analyses are included above.

$\mathrm{CI}=95 \%$ confidence internal; $n=$ number of effect sizes.

${ }^{*} p<.05 ;{ }^{* *} p<.01 ;{ }^{* * *} p<.001$. 


\section{Overall results}

Overall, the meta-analysis revealed a significant effect of sexual media on sexual outcomes (see Table 1). Concerning sexual attitudes, exposure to sexual media was positively related to permissive sexual attitudes, peer sexual norms, and acceptance of rape myths. For sexual behaviors, exposure to sexual media was positively associated with general sexual experience, and risky sexual behaviors and was negatively related to age of sexual initiation. Notably, there was no difference in the strength of effect sizes when the subcategories of attitudes, $F(2,99)=.81$, $p=.45$, or behaviors were compared, $F(2,289)=1.86, p=.16$.

\section{Heterogeneity in effect sizes}

This model allows us to examine heterogeneity both between and within studies. We found evidence of significant variation between studies $\left(\sigma^{2}=.012, \chi^{2}(1)=1627.26, p<.0001\right)$, as well as between effect sizes within studies $\left(\sigma^{2}=.007, \chi^{2}(1)=73.89, p\right.$ $<.0001)$. Sixty-three percent of the variation was within studies; $37 \%$ of the variation was between studies. Given this variation, we decided to conduct a number of moderator analyses.

\section{Moderator analyses for combined variables}

We first conducted a three-level random multiple moderator model for variables with complete data (age, study design, region, media type, transformed data, and published data). Moderators were conducted on the combined sexual outcome variable (see Note 2). Table 1 shows the major results of the moderator analysis. The analysis revealed evidence of moderation, $F(13,363)=2.92, p<.001$. The only significant moderator of sexual media effects was age of participant, $t=3.86, p<.001$, with adolescents showing significantly larger effect sizes than emerging adults. Because only one study examined the effect of sexual media on sexual outcomes in childhood, analyses were not conducted on this category. A number of interactions between variables (including different subtypes of attitudes and categories) were examined, with no evidence of any interaction between moderation being found.

Gender and ethnicity were both examined separately, given that these were reported separately in a minority of studies (see Table 1). For gender, there was evidence of moderation, $F(1$, $140)=14.58, p<.001$, with males showing larger effect sizes than females. We also examined interactions with subtypes of attitudes and behaviors. There was no interaction between gender and any sexual behavioral subtype; however, there was a significant interaction with sexual attitudes toward peers, $t=2.10, p<.05$. The interaction revealed that the effect of media on sexual attitudes toward peers was stronger for males, $r_{+}=-.12,95 \%$ confidence interval $(-.22$ to -.02$), p=.02, n=8$, compared with females, $r_{+}=-.01,95 \%$ confidence interval $(-.11$ to .08$), p=.79, n=8$.

There was also significant evidence of moderation for ethnicity, $F(2,148)=3.05, p=.05$, with effect sizes being stronger for White participants, compared with Black

Note 2:

Reference categories for each moderator are in parentheses: age (adolescence), region (North America), media type (television), transformed data (not transformed), and published data (published).

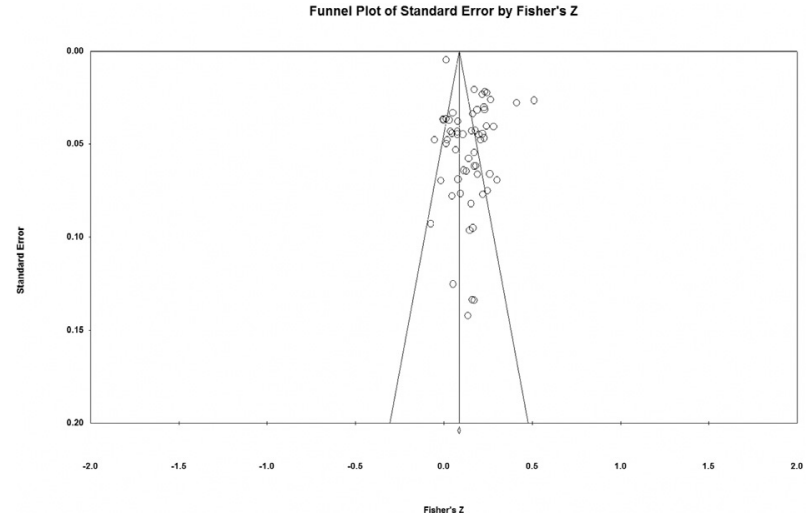

Figure 2. Funnel plot of standard error by Fisher's Z.

participants. Hispanic participants did not significantly differ from either reference category. There was no evidence of any interaction between ethnicity and subtypes of sexual behavior or attitudes.

\section{Publication bias}

Several analyses were conducted to examine potential publication bias. These analyses were all conducted using the combined sexual outcome measure. An examination of the funnel plot resulted in a largely symmetric funnel plot, suggesting that the finding is robust (see Figure 2). The fail-safe $N$ was 1,183 , suggesting that 1,183 studies showing null results would need to be unpublished in "file drawers" to reduce the overall effect to once of nonsignificance. Egger's regression test was significant, $t=3.89, p<.001$, as was the rank correlation test, $z=2.42$. $p=.01$. Finally, Duval and Tweedie's trim and fill procedure resulted in zero additional studies on the left side of the mean. Overall, these analyses suggest that the findings are robust, and publication bias in the field, although present, is fairly low.

\section{Discussion}

Overall, this meta-analysis demonstrates consistent and robust relations between media exposure and sexual attitudes and behavior spanning multiple outcome measures and multiple media. Media portray sexual behavior as highly prevalent, recreational, and relatively risk-free [3], and our analyses suggest that a viewer's own sexual decision-making may be shaped, in part, by viewing these types of portrayals. Our findings are in direct contrast with the previous meta-analysis, which suggested that media's impact on sexual behavior was trivial or nonexistent [4]. The previous meta-analysis used 38 effect sizes and found that "sexy" media were weakly and trivially related with sexual behavior $(r=.08)$, whereas the current metaanalysis used more than 10 times the amount of effect sizes ( $n=394)$ and found an effect nearly double the size $(r=.14)$.

First, we found positive associations between exposure to sexual media and teens' and young adults' permissive sexual attitudes and perceptions of their peers' sexual experiences. Second, exposure to sexual media content was associated with greater acceptance of common rape myths. Finally, sexual media exposure was found to predict sexual behaviors including age of sexual initiation, overall sexual experience, and risky sexual 
behavior. These results converged across multiple methodologies and provide support for the assertion that media contribute to the sexual experiences of young viewers.

Although the meta-analysis demonstrated significant effects of sexual media exposure on sexual attitudes and behaviors across all variables of interest, these effects were moderated by a few variables. Most notably, significant effects for all ages were apparent; however, the effect was more than twice as large for adolescents as for emerging adults, perhaps reflecting the fact that older participants likely have more comparative, real-world experience to draw on than younger participants [36,37]. In addition, the effect was stronger for males compared with females, perhaps because sexual experimentation fits the male sexual script [18] and because male characters are punished less often than female characters for sexual initiation [38]. Finally, the effect was stronger for white participants compared with black participants. This finding aligns with expectations of social cognitive theory [6], which anticipates that black youth may identify less strongly with the predominantly white characters of mainstream media.

\section{Implications}

These findings have significant implications for adolescent and emerging adult physical and mental health. Perceiving high levels of peer sexual activity and sexual permissiveness may increase feelings of internal pressure to experiment sexually [39]. In one study, exposure to sexual media content in early adolescence was seen to advance sexual initiation by 9-17 months [40]; in turn, early experimentation may increase mental and physical health risks [37].

The effect sizes found here are similar to those of other studied areas of media psychology such as media's impact on violence [41], prosocial behavior [42], and body image [43]. In each of these cases, although media use accounts for only a portion of the total variance in the outcomes of interest, media do play an important role. These comparisons suggest that sexual media content is a small, but consequential factor in the development of sexual attitudes and behaviors in adolescents and emerging adults.

\section{Limitations and future directions}

There are some limits to the strength of our findings. Our use of a deterministic imputation formula to assign standardized regression coefficients in cases where we were unable to determine $r$, although limited, is superior to replacing the missing data with either zero or the means of all the correlations [32]. However, transformed and untransformed studies showed no significant difference, suggesting this limitation is minor.

Moving forward, evidence presented here highlights the need for an ecological approach to sexual media effects that explores the unique contributions of different socialization agents (e.g., family, peers, schools, media, and religion) in predicting sexual behaviors [39]. One important aspect of this work will be a consideration of the changing digital and social media environment and its impact on viewer engagement, interpretation, and internalization of media content. In addition, we hope that research continues to examine the effects of exposure to media that contain sexual health messages, which may have a protective impact in terms of sexual risk-taking.

Adolescents and emerging adults actively seek out media to establish norms about relationships and to learn about sexuality. In the meta-analysis presented here, heightened exposure to sexual content in mainstream media has a small, but significant effect on sexual attitudes and behavior. Given the prevalence of risk-free, casual sex presented in the media and the propensity of adolescents and emerging adults to seek out these media, further determining the effects of these portrayals on viewers will be an essential area of focus for researchers, media producers, parents, and young viewers moving forward.

\section{Supplementary Data}

Supplementary data related to this article can be found at https://doi.org/10.1016/j.jadohealth.2018.11.016.

\section{References}

[1] Bleakley A, Romer D, Jamieson P. Violent film characters' portrayal of alcohol, sex, and tobacco-related behaviors. Pediatrics 2014;133:71-7.

[2] Fisher D, Hill D, Grube J, Gruber E. Sex on American television: An analysis across program genres and network types. J Broadcast Electron Media 2004;48:529-53.

[3] Kirsch A, Murnen S. 'Hot' girls and 'cool dudes': Examining the prevalence of the heterosexual script in American children's television media. Psychology of Popular Media Culture 2015;4:18-30.

[4] Ferguson C, Nielsen R, Markey P. Does sexy media promote teen sex? A meta-analytic and methodological review. Psychiatr Q 2017;88:349-58.

[5] Gerbner G, Gross L, Morgan M, et al. Growing up with television: Cultivation processes. In: Bryant J, Zillmann D, eds. LEA's communication series. Media effects: Advances in theory and research (pp. 43-67). Mahwah, NJ: Lawrence Erlbaum Associates Publishers; 2002.

[6] Bandura A. Social cognitive theory. In: van Lange PAM, Kruglanski AW, Higgins ET, eds. Handbook of Social Psychological Theories. 2011:349-73.

[7] Wright PJ. Mass media effects on youth sexual behavior: Assessing the claim for causality. Commun Yearbk 2011;35:343-86.

[8] Chia S, Lee W. Pluralistic ignorance about sex: The direct and the indirect effects of media consumption on college students' misperception of sexrelated peer norms. Int J Publ Opin Res 2008;20:52-73.

[9] Bleakley A, Hennessy M, Fishbein M, Jordan A. It works both ways: The relationship between exposure to sexual content in the media and adolescent sexual behavior. Media Psychol 2008;11:443-61.

[10] Coyne S, Padilla-Walker L. Sex, violence, \& rock n' roll: Longitudinal effects of music on aggression, sex, and prosocial behavior during adolescence. J Adolesc 2015;41:96-104.

[11] Parkes A, Wight D, Hunt K, et al. Are sexual media exposure, parental restrictions on media use and co-viewing TV and DVDs with parents and friends associated with teenagers' early sexual behaviour? J Adolesc 2013; 36:1121-33.

[12] Ward L, Epstein M, Caruthers A, Merriwether A. Men's media use, sexual cognitions, and sexual risk behavior: Testing a mediational model. Dev Psychol 2011;47:592-602.

[13] Ward L, Friedman K. Using TV as a guide: Associations between television viewing and adolescents' sexual attitudes and behavior. J Res Adolesc 2006;16:133-56.

[14] Chia S, Gunther A. How media contribute to misperceptions of social norms about sex. Mass Commun Soc 2006;9:301-20.

[15] Bond B, Drogos K. Sex on the shore: Wishful identification and parasocial relationships as mediators in the relationship between Jersey Shore exposure and emerging adults' sexual attitudes and behaviors. Media Psychol 2014;17:102-26.

[16] Chandra A, Martino S, Miu A, et al. Does watching sex on television predict teen pregnancy? Findings from a national longitudinal survey of youth Pediatrics 2008;122:1047-54.

[17] Fogel J, Kovalenko L. Reality television shows focusing on sexual relationships are associated with college students engaging in one-night stands. J Cognitive Behav Psychotherap 2013;13:321-31.

[18] Kim JL, Sorsoli CL, Collins K, et al. From sex to sexuality: Exposing the het erosexual script on primetime network television. J Sex Res 2007;44:145-57.

[19] Eaton A, Matamala A. The relationship between heteronormative beliefs and verbal sexual coercion in college students. Arch Sex Behav 2014;43: $1443-57$. 
[20] Kahlor L, Eastin MS. Television's role in the culture of violence toward women: A study of television viewing and the cultivation of rape myth acceptance in the United States. J Broadcast Electron Media 2011;55:215-31.

[21] Hust SJ, Lei M, Ren C, et al. The effects of sports media exposure on college students' rape myth beliefs and intentions to intervene in a sexual assault. Mass Commun Soc 2013;16:762-86.

[22] ter Bogt T, Engels R, Bogers S, Kloosterman M. 'Shake it baby, shake it': Media preferences, sexual attitudes and gender stereotypes among adolescents. Sex Roles 2010;63:844-59.

[23] Vandenbosch L, Eggermont S. Maternal attachment and television viewing in adolescents' sexual socialization: Differential associations across gender. Sex Roles 2012;66:38-52

[24] Brown J, L'Engle K, Pardun C, et al. Sexy media matter: Exposure to sexual content in music, movies, television, and magazines predicts black and white adolescents' sexual behavior. Pediatrics 2006;117: $1018-27$.

[25] Hennessy M, Bleakley A, Fishbein M, Jordan A. Estimating the longitudina association between adolescent sexual behavior and exposure to sexual media content. J Sex Res 2009;46:586-96.

[26] Rothstein $H$, Bushman B. Methodological and reporting errors in metaanalytic reviews make other meta-analysts angry: A commentary on Ferguson (2015). Perspect Psychol Sci 2015;10:677-9.

[27] Valkenburg P. The limited informativeness of meta-analyses of media effects. Perspect Psychol Sci 2015;10:680-2.

[28] Twenge J, Park H. The decline in adult activities among U.S. adolescents, 1976-2016. Child Dev 2017. https://doi.org/10.1111/cdev.12930.

[29] Ward L. Understanding the role of entertainment media in the sexual socialization of American youth: A review of empirical research. Dev Rev 2003;23:347-88.

[30] Ward LM, Erickson SE, Lippman J, Giaccardi S. Sexual media content and effects. In: Oxford Research Encyclopedia of Communication. New York, NY: Oxford University Press; 2016.
[31] Silver N, Dunlap W. Averaging correlation coefficients: Should Fisher's z transformation be used? J Appl Psychol 1987;72:146-8.

[32] Peterson R, Brown S. On the use of beta coefficients in meta-analysis. J Appl Psychol 2005;90:175-81.

[33] Lipsey MW, Wilson DB. Practical meta-analysis. Thousand Oaks, CA: Sage Publications, Inc; 2001.

[34] Borenstein M, Hedges L, Higgins J, Rothstein H. A basic introduction to fixed-effect and random-effects models for meta-analysis. Res Synth Methods April 2010;1:97-111.

[35] Assink M, Wibbelink CJ. Fitting three-level meta-analytic models in R: A step-by-step tutorial. Quant Methods Psychol 2016;12:154-74.

[36] Ashby S, Arcari C, Edmonson M. Television viewing and risk of sexual initiation by young adolescents. Arch Pediatr Adolesc Med 2006;160:375-80.

[37] L'Engle K, Brown J, Kenneavy K. The mass media are an important context for adolescents' sexual behavior. J Adolesc Health 2006;38:186-92.

[38] Aubrey JS. Sex and punishment: An examination of sexual consequences and the sexual double standard in teen programming. Sex Roles 2004;50: 505-14.

[39] Bleakley A, Hennessy M, Fishbein M, Jordan A. Using the integrative model to explain how exposure to sexual media content influences adolescent sexual behavior. Health Educ Behav 2011;38:530-40.

[40] Collins R, Elliott M, Berry S, et al. Watching sex on television predicts adolescent initiation of sexual behavior. Pediatrics 2004;114:e280-9.

[41] Greitemeyer T, Mügge D. Video games do affect social outcomes: A metaanalytic review of the effects of violent and prosocial video game play. Pers Soc Psychol Bull 2014;40:578-89.

[42] Coyne S, Padilla-Walker L, Hawkins A, et al. A meta-analysis of prosocial media on prosocial behavior, aggression, and empathic concern: A multidimensional approach. Dev Psychol 2018;54:331-47.

[43] Grabe S, Ward L, Hyde J. The role of the media in body image concerns among women: A meta-analysis of experimental and correlational studies. Psychol Bull 2008;134:460-76. 